\title{
Chemical Composition and Inheritance Patterns of Aroma in Fragaria $\times$ ananassa and Fragaria virginiana Progenies
}

\author{
B. Carrasco ${ }^{1}$ \\ Centro de Investigación en Biotecnología Silvoagrícola, 2 Instituto de Biología Vegetal y Biotecnología, Universidad \\ de Talca, Casilla 721, Talca, Chile \\ J.F. Hancock and R.M. Beaudry \\ Department of Horticulture, Michigan State University, East Lansing, MI 48823-1325
}

\begin{abstract}
J.B. Retamales
Facultad de Ciencias Agrarias and Centro de Investigación en Biotecnología Silvoagrícola, Universidad de Talca, Casilla 747, Talca, Chile
\end{abstract}

The strawberry aroma is one of the most popular fruit flavors (Bood and Zabetakis, 2002); its chemical composition has been extensively studied (Mc Fadden et al., 1965; Pérez et al., 2002; Schreier, 1980). To date, over 300 different aromatic compounds have been identified (Latrasse, 1991; Zabetakis and Holden, 1997). Among them, esters and furanones are considered the main aroma determinants in fresh strawberries. Although aroma is a highly appreciated organoleptic attribute, studies to determine the genetic basis of strawberry aroma are scarce (Aharoni et al., 2000). They have been focused on identifying some specific genes, but little is known about the inheritance patterns of the aroma trait and the main compounds associated with it. The objective of this study was to determine the nature of aroma inheritance in segregating populations of strawberries and identify the most important aroma components.

\section{Materials and Methods}

A diallele cross $(14 \times 14)$ was made among cultivars and elite wild selections of Fragaria $\times$ ananassa and $F$. virginiana. The progenies (174 genotypes) were planted at Michigan State University experimental farm in East Lansing. Aromas were identified in three replications of dark-red fruit on five to ten progenies of each family, with a HP 6890 gas chromatography (GC) system equipped with a fused silica Supelcowax-10 capillary column $(30 \mathrm{~m} \times 0.25 \mathrm{~mm} \times$ $0.25 \mu \mathrm{m})$. Helium $\left(1 \mathrm{~mL} \cdot \mathrm{min}^{-1}\right)$ was the carrier gas. The GC was coupled to a Leco Pegasus II mass spectrometer (MS). The identification of the main compounds was performed by comparing the mass spectra and retention time data with those of standards and using the Wiley GC-MS library. For each compound, quantification was performed by replotting the data, selecting the most abundant fragment and measuring the area under peaks. Heritability in narrow sense $\left(\mathrm{h}^{2}\right)$ was calculated for 15 compounds, using midparent-offspring regression analysis (Falconer and Mackay, 1996). Aroma compounds were screened in fruit of each of the 174 genotypes including aldehydes (2-hexenal, hexanal); alcohols(3-hexen-1-ol,(Z)); furanones (furaneol, mesifuran); esters (butyl acetate, ethyl acetate, hexyl acetate, methyl acetate, octyl acetate, 2-

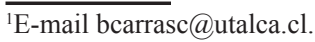

methyl ethyl butanoate, ethyl butanoate, methyl butanoate, ethyl hexanoate, and methyl hexanoate). These compounds are often mentioned in the literature as relevant for strawberry aroma (Pérez et al., 2002; Zabetakis and Holden, $1997)$ and in preliminary studies, our team found 3-hexen-1-ol, (Z), furaneol, hexyl acetate, ethyl butanoate, and ethyl hexanoate in various foods (jelly and milk) and cosmetics (lipstick) with artificial strawberry aroma. To determine the relative contribution of the compounds to strawberry aroma, the aroma value $[\mathrm{AV}=$ concentration/odor threshold (OT)] was calculated (Devos etal., 1990; Larsen and Poll, 1992; www. leffingwell.com). Additionally, a panel of 5 to 7 persons sampled aromas of fresh fruit of the 174 genotypes, maintained in glass containers for $30 \mathrm{~min}$ at room temperature. They ranked each sample into one of six categories: $6=$ superior aroma, $5=$ strong aroma, $4=$ medium aroma, 3 = moderately low aroma, $2=$ low aroma, and $1=$ no aroma. Correlation analysis determined the association between panel rank and the relative contribution of the selected aroma compounds.

To evaluate the relationships of aroma compounds diversity and panel's perception, Shannon's index (Shannon, 1948) was calculated for 72 esters and 2 furanones in a subsample of 25 genotypes previously ranked by the panel.

$$
H=-\sum_{i=1}^{S} \dot{p} \operatorname{Ln} \dot{p}
$$

In our case pi corresponded to the proportion of compound i relative to the total number of compounds by genotype.

\section{Results}

The aroma value (AV) indicates the relative impact of individual compounds to the overall aroma (Zabetakis and Holden, 1997). According to the AV level, three groups of compounds were identified in strawberry. The compounds: mesifuran, ethyl acetate, methyl butanoate, ethyl butanoate and ethyl hexanoate belong to the first group; these had the highest $\mathrm{AV}$, ranging between 1007 and 4420 (Table 1). The second group included: methyl acetate, hexyl acetate, furaneol, and 2-methyl-ethyl-butanoate whose AV ranged between 26.1 and 56.5. Finally, the third group of compounds was formed by: hexanal, 3-hexen-1ol(Z), methyl hexanoate, butyl acetate, octyl acetate and 2-hexenal, with AV between 0.007 and 4.7.

Significant correlations $(p>0.005$, Table 1) were found between panel rankings versus concentration and panel ratings versus AV for butyl acetate, hexyl acetate, 2-methyl ethyl butanoate, ethyl butanoate, methyl butanoate, ethyl hexanoate and methyl hexanoate. No significant correlation was found between furanones and panel ratings.

Large differences were found for compound abundance in the segregating population. The most aromatic samples (or genotypes) were those with highest diversity and abundance for esters. A significant correlation $(r=0.89$, $p>0.005)$ was found between Shannon's Index and how strong an aroma was perceived by the panel. The most aromatic samples (13 genotypes) according to panel ratings, had $\mathrm{H}$ values ranging from 1.71 to 2.30 and 17 to 42 compounds, while the lowest rated fruit (12 genotypes) had $\mathrm{H}=0$ to 1.10 and 0 to 11 compounds.

The aroma classifications of the panel, showed a normal distribution for the six classes analyzed. Several of the individual compounds (Table 1$)$, were significantly correlated $(P<$ $0.05)$ with the ratings of aroma intensity determined by the panel: ethyl butanoate $(r=0.53)$, ethyl hexanoate $(r=0.36)$, butyl acetate $(r=$ $0.3)$, hexyl acetate $(r=0.28)$, 2-methyl-ethyl butanoate $(r=0.28)$, 3-hexen-1-ol, (Z) $(r=$ $0.26)$, methyl butanoate $(r=0.21)$, methyl hexanoate $(r=0.2)$, octyl acetate $(r=0.19)$, and furaneol $(r=0.18)$.

The heritability (Table 1 ) was high for 2-methyl ethyl butanoate ( 0.53 ) and 3-hexen1-ol, (Z) (0.51), was medium for mesifuran (0.33), ethyl acetate $(0.33)$, 2-hexenal $(0.33)$, hexyl acetate $(0.3)$ and low for furaneol $(0.22)$, ethyl butanoate $(0.22)$, butyl acetate $(0.19)$, methyl acetate $(0.17)$, methyl butanoate $(0.12)$, ethyl hexanoate $(0.11)$, and methyl hexanoate (0.05). The compounds hexanal and octyl acetate showed zero heritability. In these samples, the heritability was low $\left(\mathrm{h}^{2}=0.04\right)$ for aroma as a trait.

\section{Discussion}

There was consistency between panel classification and chemical diversity shown by Shannon's Index. According to AV levels, some of the most important compounds associated 
Table 1. Heritability $\left(\mathrm{h}^{2}\right)$, concentration, odor threshold (OT), and aroma value (AV) for 15 aroma volatiles from dark-red strawberry fruits.

\begin{tabular}{|c|c|c|c|c|}
\hline Aroma volatiles & $h^{2 z}$ & $\begin{array}{l}\text { Concn } \\
\text { (ppm) }\end{array}$ & $\begin{array}{c}\mathrm{OT} \\
(\mathrm{ppm})^{\mathrm{y}}\end{array}$ & $\mathrm{AV}^{\mathrm{x}}$ \\
\hline \multicolumn{5}{|l|}{ Aldehydes } \\
\hline 2-Hexenal & 0.33 & 0.0007 & 0.1 & 0.007 \\
\hline Hexanal & 0 & 0.4741 & 0.1 & 4.7 \\
\hline \multicolumn{5}{|l|}{ Alcohols } \\
\hline 3-Hexen-1-ol, (Z) & 0.51 & $0.0106^{*}$ & 0.0039 & 2.7 \\
\hline \multicolumn{5}{|l|}{ Furanones } \\
\hline Furaneol & 0.22 & $0.4416^{*}$ & 0.01 & 44.2 \\
\hline Mesifuran & 0.33 & 0.0442 & 0.00001 & 4420 \\
\hline \multicolumn{5}{|l|}{ Esters } \\
\hline Butyl acetate & 0.19 & $0.0775^{*}$ & 0.06 & 1.3 \\
\hline Ethyl acetate & 0.33 & 12.8643 & 0.005 & 2573 \\
\hline Hexyl acetate & 0.30 & $0.0949^{*}$ & 0.002 & 47.5 \\
\hline Methyl acetate & 0.17 & 11.3096 & 0.2 & 56.5 \\
\hline Octyl acetate & 0 & $0.0014^{*}$ & 0.0012 & 1.2 \\
\hline 2-methyl-ethyl butanoate & 0.53 & $0.0261^{*}$ & 0.001 & 26.1 \\
\hline Ethyl butanoate & 0.22 & $1.0073^{*}$ & 0.001 & 1007 \\
\hline Methyl butanoate & 0.12 & $1.6068^{*}$ & 0.001 & 1607 \\
\hline Ethyl hexanoate & 0.11 & $0.3036^{*}$ & 0.0003 & 1012 \\
\hline Methyl hexanoate & 0.005 & $0.2542^{*}$ & 0.1 & 2.5 \\
\hline
\end{tabular}

${ }^{2} \mathrm{~h}^{2}=$ Heritability calculated for a sample size of 174 genotypes $(F$. × ananassa and $F$. virginiana $)$. Significant correlations $(p<0.05)$ between panel ratings of aroma intensity and compound concentration are indicated in the concentration column.

'Devos et al., 1990; www.leffingwell.com; Larsen and Poll, 1992.

${ }^{\mathrm{x}} \mathrm{AV}=$ concentration/OT.

"Significant correlations at $p<0.05$ between panel ratings of aroma intensity and compound concentration.

with strawberry aroma were mesifuran, ethyl acetate, methyl butanoate, ethyl hexanoate and ethyl butanoate, which agrees with previously published findings (Larsen and Poll, 1992; Pérez et al., 1992; Schreier, 1980). Moreover, we found all of these compounds in artificial strawberry aroma of some cosmetics and foods, indicating that they are important in the definition of aroma in strawberry.

Few studies have been done to determine the genetic control of aroma and its inheritance patterns in fresh fruit (Causse et al., 2001; Saliba-Colombani et al., 2001). Our results are coincident with those previously reported in other fruit, in that the aroma inheritance behaves as a complex trait.

Most aroma compounds studied showed variable heritabilities. The highest levels of heritability were shown by two compounds: 2- methyl-ethyl-butanoate $\left(\mathrm{h}^{2}=0.53\right)$ and 3hexen-1-ol $(\mathrm{Z})\left(\mathrm{h}^{2}=0.53\right)$. Probably they are involved in the basal aroma in fresh strawberry fruit. For example, it is known that 3-hexen-1-ol (Z) provides green notes to aroma for several species (Zabetakis and Holden, 1997)

Interestingly, the compounds with highest $\mathrm{AV}$ and abundance had medium to low heritabilities $(0.11$ to 0.33$)$ in the fresh fruit analyzed. This result indicates that special breeding strategies, such as family selection and hybridization methods, should be applied to improve the aroma trait in strawberries. Heritability for aroma trait as a whole appears to be low; which would indicate that under the conditions of this experiment, the aroma character as well as the production of esters and furanones, are strongly influenced by the interaction between environment and genotype.

\section{Literature Cited}

Aharoni A, L. Keizer, H. Bouwmeester, S. Zhongkui, M. Alvarez-Huerta, H. Verhoeven, J. Blaas, A. van Houwelingen, R. De Vos, H. van der Voet, R.
Jansen, M. Guis, J. Mol, R. Davis, M. Schena, A van Tunen, and O'Connell, A. 2000. Identification of the SAAT gene involved in strawberry flavor biogenesis by use of DNA microarrays. Plant Cell 12:1-16

Bood, K. and I. Zabetakis. 2002. The biosynthesis of strawberry flavor (II): Biosynthetic and molecular biology studies. J. Food Sci. 67:2-8.

Causse, M., V. Saliba-Colombani, I. Lesschaeve, and M. Buret. 2001. Genetic analysis of organoleptic quality in fresh market tomato. II. Mapping QTLs for sensory attributes. Theor.Appl. Genet. 102:273-283.

Devos M., F. Patte, J. Rouault, P. Laffort, and L.J. Van Gemert. 1990. Standarized human olfactory thresholds. IRL Press, Oxford Univ. Press, New York.

Falconer D.S. and T. Mackay. 1996. Introduction to quantitative genetics. Longman Scientific and Technical, Edinburg.

Larsen, M. and L. Poll. 1992. Odour thresholds of some important compounds in strawberries. Zeitschrift für lebensmittel-untersuchung und-forschung 195:120-123.

Latrasse, S. 1991. Fruits III, p. 329-387. In: H. Maarse (ed.). Volatile compounds in foods and beverages. Marcel-Dekker, New York.

McFadden, W., R. Teranishi, J. Corse, O. Black, and T. Mon. 1965. Volatiles from strawberries II. Combined mass spectrometry and gas chromatography on complex mixture. J. Chromatog. 18:10-19.

Pérez, A., J. Rios, C. Sanz, and R. Olias. 1992. Aroma components and free aminoacids in strawberry variety Chandler during ripening. J. Agr. Food Chem. 40:2232-2235.

Pérez, A., R. Olias, P. Luaces, and C. Sanz. 2002. Biosynthesis of strawberry aroma compounds through amino acid metabolism. J. Agr. Food Chem. 50:4037-4042.

Saliba-Colombani, V., M. Causse, D. Langlois, J. Philouse, and M. Buret. 2001. Genetic analysis of organoleptic quality in fresh market tomato. I. Mapping QTLs for physical and chemical traits. TAG 102:259-272.

Schreier, P. 1980. Quantitative composition of volatile constituents in cultivated strawberries, Fragaria ananassa cvs. Senga Sengana, Senga Litessa and Senga Gourmella. J. Sci. Food Agr. 31: $487-494$

Shannon, C. 1948. A mathematical theory of communication. Bell System Tech. J. 27 379-423.

Zabetakis, I. and M. Holden. 1997. Strawberry flavour: analysis and biosynthesis. J. Sci. Food Agr. 74:421-434. 